\title{
Análisis de la percepción de los residentes sobre el impacto cultural y deportivo de un Gran Premio de Fórmula 1
}

\author{
Ferran Calabuig Moreno* \\ David Parra Camacho** \\ Vicente Añó Sanz**** \\ Daniel Ayora Pérez ${ }^{* * * *}$
}

\begin{abstract}
Resumen: El objetivo de este estudio es analizar la percepción de los residentes sobre el impacto cultural y deportivo del Gran Premio de Europa de Fórmula 1. Se realizó un análisis clúster, combinando métodos jerárquicos y no jerárquicos, que permitió encontrar tres grupos con percepciones diferentes hacia este evento deportivo: Desfavorables, Desfavorables Moderados y Favorables Moderados. Los residentes consideran de forma mayoritaria que este evento no tiene un impacto considerable a nivel cultural y deportivo. Asimismo, se encontraron diferencias entre los tres grupos de residentes en función del interés por la Fórmula 1 , el respaldo al evento, la asistencia y el grado de identificación. Palabras clave: Población residente. Percepción social. Deportes. Factores culturales.
\end{abstract}

\footnotetext{
DDepartment of Physical Education and Sports. Facultad de Ciencias de la Actividad Física y el Deporte. Universitat de València. Valencia, Espanha. E-mail: ferran.calabuig@uv.es

"Department of Physical Education and Sports. Facultad de Ciencias de la Actividad Física y el Deporte. Universitat de València. Valencia, Espanha. E-mail: david.parra-camacho@uv.es

".'Department of Physical Education and Sports. Facultad de Ciencias de la Actividad Física y el Deporte. Universitat de València. Valencia, Espanha. E-mail: vicent.anyo@uv.es

...'Department of Physical Education and Sports. Facultad de Ciencias de la Actividad Física y el Deporte. Universitat de València. Valencia, Espanha. E-mail: daniel.ayora@uv.es
} 


\section{INTRODUCCIÓN}

Los eventos deportivos repercuten en la vida de los residentes de las localidades o regiones en las que se celebran. Así, podemos encontrar impactos positivos y negativos que están asociados a la acogida de estos acontecimientos y que pueden influir en la calidad de vida de los residentes. Diversos autores han clasificado y recopilado los posibles impactos de los eventos deportivos (FREDLINE, 2004; PREUSS; SOLBERG, 2006; TAVARES, 2011). Tradicionalmente, el interés de la administración y los organizadores se ha centrado en el análisis del impacto económico debido a la necesidad de justificar la rentabilidad de las inversiones realizadas en el acontecimiento. Sin embargo, en los eventos deportivos existen diversos grupos de interés o stakeholders que hacen necesaria una evaluación más amplia y exhaustiva de su repercusión en la comunidad de acogida. Por esta razón, cada vez existe un reconocimiento más unánime entre los investigadores y los organizadores de la necesidad de medir los impactos socio-culturales de los mismos, ya que la insatisfacción de la comunidad de acogida puede poner en peligro su éxito a largo plazo aunque el acontecimiento sea viable a nivel económico (SMALL; EDWARDS; SHERIDAN, 2005).

Numerosos estudios han analizado el impacto social de los eventos deportivos destacando sobre todo las aportaciones realizadas sobre grandes o mega eventos deportivos como los Juegos Olímpicos (MIHALIK; SIMONETTA, 1999; WAITT, 2003; ZHOU; AP, 2009) y mundiales de Fútbol (KIM; PETRICK, 2005; KIM, GURSOY; LEE, 2006; OHMANN; JONES; WILKES, 2006).

En este estudio se muestran los resultados de las percepciones de los residentes sobre una prueba del Campeonato del Mundo de Fórmula 1, concretamente el Gran Premio (GP) de Europa de Fórmula 1. No obstante, en este trabajo nos centraremos en el análisis de las percepciones de los ciudadanos sobre el impacto cultural y deportivo que este acontecimiento ha supuesto para la ciudad de Valencia, localidad en la que se celebra este evento anualmente desde 
su incorporación al calendario de pruebas en 2008. Muchos estudios que analizan la opinión pública sobre los eventos deportivos incluyen aspectos relacionados con el impacto cultural y deportivo reflejados en dimensiones específicas como el estudio de Kim, Gursoy y Lee. (2006) (beneficios de intercambio cultural), o como el de Balduck, Maes y Buelens (2011) que contemplan un factor relacionado con el interés y la consolidación cultural. En cuanto a los aspectos relacionados con el impacto deportivo suelen quedar englobados en otras dimensiones como el impacto socio-cultural o los beneficios para la comunidad.

Es muy frecuente encontrar alusiones de las autoridades $\mathrm{u}$ organizadores de grandes eventos deportivos en torno a la posibilidad de fomentar el desarrollo deportivo de las regiones como consecuencia de la acogida de estos acontecimientos. No obstante, diversos estudios no consideran que se produzcan importantes incrementos en los niveles de práctica deportiva (COALTER; TAYLOR, 2009; WEED; COREN; FIORE, 2009).

Por otro lado, a la falta de evidencia clara de que la celebración de eventos deportivos incremente los niveles de práctica de actividad física se unen las características particulares de la Fórmula 1, ya que se trata de un deporte de motor cuyo acceso es muy reducido para la población debido a sus elevados costes y la dificultad para poder practicarlo con seguridad.

Estudios como los de Fredline y Faulkner (2000) sobre la Gold Coast IndyCar Race, el de Fredline (2004) y Fredline, Deery y Jago (2005) sobre el GP de Australia, el de Cheng y Jarvis (2010) sobre el GP de Fórmula 1 de Singapur, el de Zhou (2010) sobre el GP de Fórmula 3 de Macao o el realizado por Añó, Calabuig y Parra (2012) sobre la Fórmula 1, han consultado la percepción de los residentes sobre el impacto social de estos acontecimientos. En los trabajos de Fredline y Faulkner (2000), Fredline (2004) y Fredline, Deery y Jago (2005), que agrupan a los ciudadanos mediante un análisis clúster según sus percepciones sobre los impactos del evento, destacan que los residentes con reacciones más negativas hacia el mismo tienden 
a mostrarse en desacuerdo ante posibles impactos positivos para la comunidad derivados de la acogida de la Fórmula 1, mientras que los ciudadanos con percepciones más favorables muestran la tendencia inversa. En los aspectos consultados en el estudio de Fredline y Faulkner (2000) sobre el impacto cultural y deportivo, podemos destacar que la mayoría de los residentes se mostraban de acuerdo en considerar la celebración de la IndyCar como una oportunidad para asistir a un evento internacional. Esta tendencia era similar en los grupos de ciudadanos con una actitud más favorable al acontecimiento para los aspectos relacionados con la mejora de la hospitalidad de los residentes y la oportunidad para conocer nuevas personas. Sin embargo, la mayoría de colectivos no se mostraban de acuerdo, excepto los más favorables al evento, ante la posibilidad de que este acontecimiento permitiese promocionar los deportes de motor en Australia o dar nuevas oportunidades a los jóvenes pilotos y, de forma más acusada, ante la posibilidad de que se desarrollasen nuevas infraestructuras que pudiesen ser aprovechadas después por los residentes.

En el estudio de Cheng y Jarvis (2010) se observó que la promoción del intercambio cultural y la interacción, así como, la oportunidad para conocer nuevas personas eran los aspectos más mencionados como impactos socio-culturales positivos por los residentes en Singapur, mientras que la promoción del deporte eran los menos citados. En este estudio, la mayoría de ciudadanos consultados consideraban que la acogida del GP de Fórmula 1 demostraba la capacidad de los singapurenses a la hora de organizar eventos deportivos de carácter internacional e incrementaba las oportunidades de entretenimiento antes, durante y después del evento. Sin embargo, no consideraban de forma tan clara que la Fórmula 1 les diese la posibilidad para aprender más sobre otras culturas o de disfrutar conociendo otras personas.

En el caso del estudio de Zhou (2010) y de forma similar a lo detectado en el estudio de Fredline y Faulkner (2000) la mayoría de residentes consideraba que este evento ofrecía a los ciudadanos una oportunidad para asistir a un acontecimiento internacional. 
Además, opinaban que la celebración de la Fórmula 3 en Macao ayudaría a los ciudadanos a entender y conocer a otras personas y culturas. Por el contrario, desde el punto de vista de la repercusión deportiva, los residentes no se mostraban de acuerdo ante la posibilidad de que este evento deportivo proporcionase instalaciones que después podrían ser utilizadas por la comunidad local.

Por último, el estudio de Añó, Calabuig y Parra (2012) contempla la repercusión deportiva de la Fórmula 1 a través de varios ítems: incremento de la práctica deportiva en la ciudad, incremento de las instalaciones deportivas, aumento del prestigio deportivo, incremento del interés por el deporte y la Fórmula 1 está pensada para el disfrute de los ciudadanos. Los autores concluyen que los residentes manifestaron su desacuerdo ante la posibilidad de que este evento deportivo incremente la práctica deportiva o aumente las instalaciones deportivas.

No obstante, dicho estudio no recoge información sobre la percepción de los residentes sobre el impacto cultural y sobre otros aspectos relacionados con el impacto deportivo a los que hacen referencia otros trabajos mencionados como, por ejemplo, la posibilidad de promocionar el deporte o dar nuevas oportunidades a los jóvenes deportistas, la capacidad de organizar un evento deportivo, el incremento de subvenciones o apoyo a los clubes deportivos. Por esta razón, y partiendo de la revisión de los estudios analizados (AÑÓ; CALABUIG; PARRA 2012; CHENG; JARVIS, 2010; FREDLINE, 2004; FREDLINE; FAULKNER, 2000; ZHOU, 2010) se ha realizado una recopilación de aspectos y se han plateado una serie de ítems relacionados con el impacto cultural y deportivo de los eventos deportivos de motor que quedan reflejados en la tabla 1. 
Tabla 1. Impacto cultural y deportivo de los eventos deportivos de motor.

\begin{tabular}{|c|c|}
\hline Impacto Cultural & Impacto Deportivo \\
\hline $\begin{array}{l}\text { Proporciona la oportunidad para conocer } \\
\text { nuevas personas }\end{array}$ & $\begin{array}{l}\text { Incrementa la práctica deportiva de los } \\
\text { ciudadanos }\end{array}$ \\
\hline $\begin{array}{l}\text { Mejora la solidaridad y la hospitalidad de } \\
\text { los residentes con los visitantes }\end{array}$ & $\begin{array}{l}\text { Proporciona nuevas instalaciones } \\
\text { deportivas }\end{array}$ \\
\hline $\begin{array}{l}\text { Favorece el intercambio cultural y la } \\
\text { comprensión de otras culturas }\end{array}$ & $\begin{array}{l}\text { Promociona los deportes de motor y da } \\
\text { nuevas oportunidades a los jóvenes } \\
\text { deportistas }\end{array}$ \\
\hline $\begin{array}{l}\text { Favorece la preservación y conservación } \\
\text { de la cultura local }\end{array}$ & Incrementa el interés por la Fórmula 1 \\
\hline $\begin{array}{l}\text { Incrementa la cohesión y la unidad de la } \\
\text { sociedad }\end{array}$ & $\begin{array}{l}\text { Proporciona apoyo deportivo y } \\
\text { subvenciones a los clubes de la ciudad }\end{array}$ \\
\hline $\begin{array}{l}\text { Proporciona a los ciudadanos una } \\
\text { oportunidad para asistir a un evento } \\
\text { internacional }\end{array}$ & $\begin{array}{l}\text { Permite mostrar la capacidad de la } \\
\text { sociedad para acoger grandes eventos } \\
\text { deportivos }\end{array}$ \\
\hline \multirow[t]{2}{*}{$\begin{array}{l}\text { Hace que la vida nocturna sea más } \\
\text { emocionante y entretenida }\end{array}$} & Incrementa el prestigio deportivo \\
\hline & $\begin{array}{l}\text { Proporciona a los ciudadanos una } \\
\text { oportunidad de participar en la } \\
\text { organización de grandes eventos } \\
\text { deportivos }\end{array}$ \\
\hline
\end{tabular}

Fuente: Elaboración propia a partir de Añó; Calabuig; Parra (2012); Cheng y Jarvis (2010); Fredline y Faulkner (2000), Fredline (2004) y Zhou (2010).

Así, pues, en este estudio analizaremos la percepción de los residentes sobre los aspectos resumidos en la tabla 1, estableceremos grupos de ciudadanos con percepciones similares sobre el impacto cultural y deportivo del GP de Europa de Fórmula 1 en Valencia y definiremos las características de su perfil.

\section{MÉTodo}

\subsection{MuestRA Y PROCEDIMIENTO}

Un total de 442 residentes en Valencia completaron la encuesta realizada. En el momento de realizar la entrevista se preguntó a los ciudadanos si residían de forma permanente o por un tiempo prolongado (por motivos académicos, laborales, etc.) con el objetivo 
de evitar a los visitantes o personas que no residiesen en la ciudad. Se optó por aplicar un método de muestreo de conveniencia debido a la ausencia de un marco de muestreo adecuado y de un censo de residentes permanentes en la ciudad de Valencia, diferente de empadronados. No obstante, los entrevistadores fueron entrenados para administrar las encuestas mediante un seminario de formación y se les indicó que recogieran encuestas de distintos grupos de población para lograr el mayor equilibrio posible en diversas variables sociodemográficas relacionadas con el sexo o la edad.

\subsection{INSTRUMENTO}

La elaboración del cuestionario se realizó a partir de diversos estudios previos sobre el impacto social de los eventos deportivos (AÑó; CALABUIG; PARRA., 2012; BALDUCK; MAES; BUELENS, 2011; CHENG; JARVIS, 2010; FREDLINE; FAULKNER, 2000; KIM; GURSOY; LEE., 2006; ZHOU; AP, 2009; ZHOU, 2010) y sobre otros festivales o acontecimientos culturales (DELAMERE, 2001; SMALL; EDWARDS; SHERIDAN, 2005), haciendo especial hincapié en los aspectos que recogían la información relacionada con el impacto cultural y deportivo.

La encuesta está formada por un total de 15 ítems de respuesta alternativa en una escala Likert de cinco puntos. Además se incluyen diversas preguntas sobre el interés por la Fórmula 1, el respaldo al evento, la asistencia al acontecimiento y el grado de identificación, así como, cuestiones relacionadas con las variables sociodemográficas. Estas variables intrínsecas permitirán definir las características de los grupos de residentes con percepciones similares hacia el evento, así como, validar la solución clúster.

\subsection{ANÁLISIS DE LOS DATOS}

A partir de los datos de las encuestas se realizó un análisis clúster, con el programa estadístico SPSS versión 20.0, con los 15 ítems que recogían la percepción de los residentes sobre los impactos 
culturales y deportivos del evento deportivo. Siguiendo las recomendaciones de Hair, Anderson, Tatham y Black (1999) se combinaron dos métodos de estimación (jerárquicos y no jerárquicos) de la solución clúster con la finalidad de optimizar los resultados. El análisis clúster jerárquico se realizó utilizando el proceso de agrupación del Método de Ward y como medida de similaridad la distancia euclídea al cuadrado, obteniendo el historial de aglomeración. A partir de los grupos propuestos en el análisis anterior se aplicó un análisis no jerárquico a través del método de K-medias utilizando como centros iniciales o puntos de semilla las medias de las variables obtenidas para cada solución clúster del análisis jerárquico.

Después, se analizaron las diferencias entre las variables incluidas en el análisis de los clústers a partir de los valores de la prueba del ANOVA, teniendo en cuenta la advertencia de Hair et al. (1999) sobre la necesidad de que los datos de esta prueba deben utilizarse con precaución y con una finalidad descriptiva. Para definir las características de los perfiles de los grupos y para evaluar la validez predictiva se realizaron ANOVAS y pruebas Chi-Cuadrado con variables que no fueron incluidas en el análisis inicial.

\section{Resultados}

Al analizar los resultados del análisis clúster jerárquico encontramos diversas soluciones mediante la observación del historial de aglomeración. Por otro lado, la mayoría de aportaciones realizadas en este ámbito señalan soluciones de dos, tres o cinco grupos. Por esta razón, se decidió someter en el segundo análisis las posibles soluciones (dos, tres, cuatro y cinco grupos) a través del método de k-medias. Teniendo en cuenta estos resultados y los estudios previos se decidió escoger la solución de tres clúster ya que permitía ofrecer una buena interpretación de los resultados.

Las variables que más distinguen a los grupos son "creo que gracias la F1 ha aumentado la práctica deportiva de los ciudadanos" ( $f=287.93$ ), seguida de "gracias a la F1 han aumentado las 
instalaciones deportivas" ( $\mathrm{f}=233,79)$ y "mi interés por la F1 ha aumentado gracias a su celebración en mi ciudad" ( $f=215,65$ ), mientras que la que menos diferencia a los grupos es la relacionada con "la F1 proporciona a los ciudadanos una oportunidad para asistir a un evento internacional." ( $f=59,45)$.

Como podemos observar en la tabla 2, el clúster uno representa el $42,1 \%$ de la muestra, cuyas puntuaciones sobre los diversos ítems relacionados con el impacto cultural y deportivo del GP de Europa de Fórmula 1 son bastante reducidas. Por tanto, este grupo ha sido denominado como Desfavorables, ya que manifiestan una actitud desfavorable hacia la repercusión cultural y deportiva de este acontecimiento. La mayoría de las medias otorgadas por este colectivo presentan valores inferiores en la escala Likert a dos (en desacuerdo) y cercanas a uno (totalmente en desacuerdo). Por ejemplo, estas puntuaciones se observan en los ítems referidos a creo que ha incrementado la práctica deportiva de los ciudadanos $(1,24)$, han aumentado las instalaciones deportivas $(1,31)$, han incrementado las subvenciones y el apoyo deportivo a los clubes de la ciudad $(1,33)$ o la Fórmula 1 está pensada para el entretenimiento de los ciudadanos $(1,30)$. Sólo el ítem relacionado con la posibilidad de asistir a un evento internacional muestra una media superior a dos $(2,55)$. En algunos estudios los grupos de residentes con valores tan extremos suelen denominarse, en este caso, como "enemigos" del evento (FREDLINE; FAULKNER, 2000), ya que manifiestan una clara oposición a la celebración del acontecimiento.

El grupo dos representa el 33,9\% de la muestra, que reciben la denominación de Desfavorables Moderados, ya que la mayoría de aspectos consultados muestran valores que oscilan entre dos y tres en la escala Likert. De este modo, tal y como podemos comprobar en la tabla 2 , los ítems con medias más reducidas coinciden en algunos casos con los del grupo de residentes Desfavorables (e.g. incremento de la práctica deportiva, incremento de las instalaciones deportivas, subvenciones y apoyo deportivo a los clubes, está pensada para el entretenimiento de los ciudadanos). Sin embargo, algunos aspectos 
son puntuados con medias superiores a tres que indicarían cierto grado de acuerdo ante aspectos como los referidos a la posibilidad de asistir a un evento internacional $(3,67)$ o el hecho de que este acontecimiento incrementa el prestigio deportivo de España $(3,27)$. Por tanto, aunque este grupo no presenta una actitud tan desfavorable hacia la Fórmula 1 como el primer grupo, manifiesta cierto desacuerdo en la evaluación de los impactos culturales y deportivos que ha generado.

Por último, el tercer grupo representa el $24 \%$ de la muestra y podría recibir el nombre de Favorables Moderados con respecto a la acogida del GP de Europa de Fórmula 1. Esta denominación se puede entender por el hecho de que muchos de los ítems obtienen valoraciones cercanas a cuatro (de acuerdo), aunque otros aspectos muestran valores cercanos a tres, lo que señala una actitud neutral hacia diversos ítems consultados. Por ejemplo, este grupo muestra una tendencia al acuerdo en aspectos como la oportunidad de asistir a un evento internacional $(3,86)$, la mejora del prestigio deportivo $(3,82)$ y la posibilidad de que este evento promocione los deportes de motor y de nuevas oportunidades a los jóvenes pilotos $(3,68)$. No obstante, ningún ítem obtiene valores superiores a cuatro, de ahí, que no se pueda denominar a este colectivo como favorables, aficionados $\mathrm{u}$ optimistas con respecto a la celebración de este acontecimiento. 
Tabla 2. Valor medio para cada variable en los tres clústers y las pruebas $\mathrm{F}$ de las variables entre los tres clústers (obtenidos a través del método de K-medias)

\begin{tabular}{|c|c|c|c|c|c|}
\hline & $\begin{array}{c}1 \\
\text { Detractores } \\
(\mathrm{n}=186)\end{array}$ & $\begin{array}{c}2 \\
\text { Desfavorables } \\
\text { moderados } \\
(\mathrm{n}=150)\end{array}$ & $\begin{array}{l}3 \\
\text { Favorables } \\
\text { Moderados } \\
(\mathrm{n}=106)\end{array}$ & $\mathrm{F}$ & $\mathrm{P}$ valor \\
\hline $\begin{array}{l}\text { Creo que gracias a la F1 ha } \\
\text { aumentado la práctica deportiva de } \\
\text { los ciudadanos. }\end{array}$ & 1,24 & 1,87 & 3,39 & 287,93 & $.000^{* * * *}$ \\
\hline $\begin{array}{l}\text { Gracias a la F1 han aumentado las } \\
\text { instalaciones deportivas. }\end{array}$ & 1,31 & 1,88 & 3,35 & 233,79 & $.000^{\text {*** * }}$ \\
\hline $\begin{array}{l}\text { La F1 ha incrementado las } \\
\text { subvenciones y el apoyo deportivo a } \\
\text { los clubes de la ciudad. }\end{array}$ & 1,33 & 2,03 & 2,99 & 144,49 & $.000^{* * * *}$ \\
\hline $\begin{array}{l}\text { La F1 promociona los deportes de } \\
\text { motor en España y da nuevas } \\
\text { op ortunidades a los pilotos jóvenes. }\end{array}$ & 1,90 & 2,79 & 3,68 & 117,21 & $.000^{* * * *}$ \\
\hline $\begin{array}{l}\text { La F1 proporciona a los ciudadanos } \\
\text { la oportunidad de participar en la } \\
\text { organización de grandes eventos } \\
\text { deportivos. }\end{array}$ & 1,60 & 2,71 & 3,62 & 157,82 & $.000^{* * * *}$ \\
\hline $\begin{array}{l}\text { Mi interés por la F1 ha aumentado } \\
\text { gracias a su celebración en mi } \\
\text { ciudad. }\end{array}$ & 1,38 & 2,05 & 3,68 & 215,65 & $.000^{* * * *}$ \\
\hline $\begin{array}{l}\text { La F1 aumenta el prestigio } \\
\text { deportivo de España. }\end{array}$ & 2,03 & 3,27 & 3,82 & 126,18 & $.000^{* * * *}$ \\
\hline $\begin{array}{l}\text { La F1 está pensada para el } \\
\text { entretenimiento de los ciudadanos. }\end{array}$ & 1,30 & 1,89 & 3,43 & 202,82 & $.000^{* * * *}$ \\
\hline $\begin{array}{l}\text { La F1 proporciona a los ciudadanos } \\
\text { una oportunidad para asistir a un } \\
\text { evento internacional. }\end{array}$ & 2,55 & 3,63 & 3,86 & 59,45 & $.000^{* * * * *}$ \\
\hline $\begin{array}{l}\text { La F1 aumenta la cohesión y la } \\
\text { unidad de la sociedad. }\end{array}$ & 1,39 & 2,25 & 3,19 & 170,08 & $.000^{\text {**** }}$ \\
\hline $\begin{array}{l}\text { La F1 proporciona a los ciudadanos } \\
\text { una oportunidad para conocer a } \\
\text { nuevas personas. }\end{array}$ & 1,73 & 3,02 & 3,50 & 172,26 & $.000^{* * * *}$ \\
\hline $\begin{array}{l}\text { La F1 mejora la solidaridad y la } \\
\text { hospitalidad de los ciudadanos con } \\
\text { los visitantes. }\end{array}$ & 1,54 & 2,65 & 3,48 & 191,50 & $.000^{* * * *}$ \\
\hline $\begin{array}{l}\text { La F1 favorece el intercambio } \\
\text { cultural y la comprensión de otras } \\
\text { culturas. }\end{array}$ & 1,73 & 2,62 & 3,62 & 165,75 & $.000^{* * * *}$ \\
\hline $\begin{array}{l}\text { La F1 favorece la preservación y } \\
\text { conservación de la cultura } \\
\text { valenciana. }\end{array}$ & 1,27 & 2,00 & 3,30 & 212,05 & $.000^{\text {**** }}$ \\
\hline $\begin{array}{l}\text { La F1 hace que la vida nocturna sea } \\
\text { más emocionante y entretenida. }\end{array}$ & 1,55 & 2,49 & 3,68 & 157,67 & $.000^{* * * *}$ \\
\hline
\end{tabular}

Nota: Los valores de las medias se basan en una escala de tipo Likert de 5 puntos (1: totalmente en desacuerdo; 3: ni de acuerdo ni en desacuerdo; 5: totalmente de acuerdo).

***p $? 0.001$ 
Por otro lado, el perfil de los residentes que componen cada clúster se obtiene a partir de otras variables adicionales que, además, permiten asegurar la validez predictiva de los grupos propuestos. De esta forma, en la tabla 3 se muestran las variables utilizadas para definir dicho perfil en función de su percepción sobre los impactos culturales y deportivos de la Fórmula 1 en Valencia.

Así, podemos comprobar cómo el grupo de residentes desfavorables al evento deportivo son los que menos interés muestran por este deporte (un 47,8\% no le interesa la Fórmula 1), los que menos respaldan la celebración del acontecimiento $(74,7 \%)$ y la mayoría no han asistido al acontecimiento $(85,5 \%)$. Además, al ser consultados por diferentes aspectos relacionados con el grado de identificación con el evento muestran valores reducidos, manifestando su desacuerdo o total desacuerdo en aspectos como la recomendación de asistir, hablar a favor de la Fórmula 1 o considerar que este evento es beneficioso para los ciudadanos. En cuanto al perfil sociodemográfico hay que destacar la mayor proporción de mujeres al ser comparados con los otros grupos, así como, el elevado porcentaje de residentes que llevan residiendo en la ciudad más de diez años.

El grupo de residentes desfavorables moderados, de forma diferente al grupo de desfavorables, manifiesta más interés en este deporte $(45,3 \%$ sí le interesa la F1), no respalda la acogida de este evento deportivo aunque con porcentajes inferiores al grupo anterior $(40 \%)$ y la mayoría tampoco ha asistido al evento $(81,2 \%)$. No obstante, como podemos observar en la tabla 3, presentan medias más elevadas en los aspectos relacionados con el grado de identificación que el grupo de desfavorables aunque muestran una tendencia al desacuerdo en los tres ítems consultados. Por último, tienen una edad media similar al resto de grupos, con un ligero incremento en la proporción de hombres con respectos al grupo de residentes más pesimistas y en su mayoría llevan residiendo en la ciudad más de diez años.

Por último, el grupo de residentes denominado como Favorables Moderados se desmarca de forma clara de los otros dos colectivos, 
manifestando un mayor interés por este deporte $(72,1 \%$ si muestra interés por la Fórmula 1), elevado apoyo a la celebración del acontecimiento $(72,4 \%$ ) y un incremento en el porcentaje de personas de este grupo que ha asistido al evento $(43,4 \%)$. También, muestra una tendencia positiva en el grado de identificación con el acontecimiento, sobre todo en los aspectos relacionados con la recomendación de asistencia al evento y el intercambio de impresiones favorables a la celebración de la Fórmula 1. Respecto a las características sociodemográficas hay que destacar el incremento en la proporción de hombres de este grupo al compararlo con los otros, sobre todo con el de residentes desfavorables.

Tabla 3. Perfiles de los distintos grupos (clústers)

\begin{tabular}{|c|c|c|c|c|}
\hline Variable & Alternativa de respuesta & $\begin{array}{c}1 \\
\text { Desfa vorables } \\
(\mathrm{n}=186)\end{array}$ & $\begin{array}{c}2 \\
\text { Desfavorables } \\
\text { Moderados } \\
(\mathrm{n}=150)\end{array}$ & $\begin{array}{c}3 \\
\text { Favorables } \\
\text { Moderados } \\
(\mathrm{n}=106)\end{array}$ \\
\hline \multirow{3}{*}{$\begin{array}{l}\text { Interés por la } \mathbf{F} 1^{* * *} \\
\chi_{(4)}^{2}=66,95, p<0,001\end{array}$} & Sí & $25,8 \%$ & $45,3 \%{ }^{(1)}$ & $72,1 \%{ }^{(1)(2)}$ \\
\hline & No & $47,8 \%^{(2)(3)}$ & $25,3 \%$ & $13,5 \%$ \\
\hline & Indiferente & $26,3 \%$ & $29,3 \%{ }^{(3)}$ & $14,4 \%$ \\
\hline \multirow{3}{*}{$\begin{array}{l}\text { Apoyo a la } \\
\text { celebración del } \\
\text { evento } \\
\chi_{(4)}^{2}=157,89, \mathrm{p}<0,001 \\
\end{array}$} & Sí & $8,6 \%$ & $27,3 \% \%^{(1)}$ & $72,4 \%^{(1)(2)}$ \\
\hline & No & $74,7 \%^{(2)(3)}$ & $40,0 \%{ }^{(3)}$ & $16,2 \%$ \\
\hline & Indiferente & $16,7 \%$ & $32,7 \% \%^{(1)(3)}$ & $11,4 \%$ \\
\hline \multirow{2}{*}{$\begin{array}{l}\text { Asistencia al } \\
\text { evento*** } \\
\chi_{(2)}^{2}=32,77, \mathrm{p}<0,001 \\
\end{array}$} & Sí & $14,5 \%$ & $18,8 \%$ & $43,4 \%^{(1)(2)}$ \\
\hline & No & $85,5 \%^{(3)}$ & $81,2 \%{ }^{(3)}$ & $56,6 \%$ \\
\hline \multirow{3}{*}{$\begin{array}{l}\text { Grado de } \\
\text { identificación con el } \\
\text { evento }\end{array}$} & $\begin{array}{l}\text { En las conversaciones que } \\
\text { tengo hablo a favor de la } \\
\mathrm{F} 1 . \mathrm{F}_{(2)}=140,408, p>0,001\end{array}$ & 1,64 & 2,54 & 3,86 \\
\hline & $\begin{array}{l}\text { Si alguien me pide opinión, } \\
\text { recomendaré que asista a la } \\
\mathrm{F} 1 \text { como espectador. } \\
\mathrm{F}_{(2)}=133,619, p>0,001\end{array}$ & 1,63 & 2,69 & 3,81 \\
\hline & $\begin{array}{l}\text { En general, creo que la } \\
\text { inversión en F1 es } \\
\text { beneficiosa para los } \\
\text { ciudadanos. } \\
\mathrm{F}_{(2)}=137,598, p>0,001\end{array}$ & 1,42 & 2,16 & 3,56 \\
\hline \multirow{2}{*}{$\begin{array}{l}\text { Sexo } \\
\chi_{(2)}^{2}=8,34, p>0,05\end{array}$} & Hombre & $50,5 \%$ & $57,3 \%$ & $67,9 \% \%^{(1)}$ \\
\hline & Mujer & $49,5 \%^{(3)}$ & $42,7 \%$ & $32,1 \%$ \\
\hline $\begin{array}{l}\text { Edad } \\
\mathrm{F}_{(2)}=.926, \mathrm{p}>0,05\end{array}$ & Media de edad & 33,26 & 35,05 & 32,64 \\
\hline \multirow{4}{*}{$\begin{array}{l}\text { Tiempo residiendo } \\
\text { en Valencia } \\
\chi_{(6)}^{2}=14,82, p>0,05\end{array}$} & Menos de 1 año & $1,1 \%$ & $6,0 \%^{(1)}$ & $4,0 \%$ \\
\hline & De 1 a 3 años & $9,7 \%$ & $10,0 \%$ & $16,0 \%$ \\
\hline & De 4 a 10 años & $9,2 \%$ & $16,7 \%$ & $14,0 \%$ \\
\hline & Más de 10 años & $80,0 \%^{(2)(3)}$ & $67,3 \%$ & $66,0 \%$ \\
\hline
\end{tabular}


Nota. *Indica relación estadísticamente significativa o diferencias de medias estadísticamente significativas *p<0,05; **p? 0,01 ; ***p? 0,001 ; (1) (2) (3) Los resultados se basan en pruebas bilaterales con un nivel de significación 0.05. En la tabla de resultados se muestra para cada par significativo la clave del grupo de residentes con la proporción de columna menor debajo del grupo de residentes con mayor proporción de columna.

\section{DisCUSIÓN Y CONCLUSIONES}

En general es de destacar el elevado número de residentes que se muestran en desacuerdo en torno a los aspectos culturales y deportivos consultados. En el caso de los desfavorables al acontecimiento por su evidente pesimismo o tendencia al desacuerdo, podemos comprobar que se trata del grupo más numeroso $(42,1 \%)$ y su oposición a la celebración del evento es bastante elevada. Este colectivo coincide con lo detectado en otros estudios en los que ha sido denominado como Haters o "enemigos del evento" (FREDLINE; FAULKNER, 2000). Estos autores destacan que un 65\% de los residentes de este grupo no apoyaban la celebración de la IndyCar en GoldCoast (Australia), algo inferior al porcentaje de ciudadanos que no apoyaban la celebración en este trabajo $(74,7 \%)$. Además, estos investigadores destacaron que los residentes de este grupo no consideraban que este evento proporcionase instalaciones que después pudiesen ser utilizadas por los ciudadanos, hecho que coincide con la tendencia al desacuerdo detectada en este trabajo. Sin embargo, mostraron una percepción más neutral, diferente a la de este estudio, en torno a la posibilidad de promocionar los deportes de motor y dar nuevas oportunidades a los jóvenes pilotos.

En una posición intermedia, aunque también con una tendencia al desacuerdo en muchos aspectos, se encontraría el grupo de desfavorables moderados. Este colectivo se opone de forma mayoritaria o se muestra indiferente a la celebración del evento y considera que el impacto cultural y deportivo del mismo es bastante reducido. Sin embargo, muestran una tendencia neutral en algunos aspectos como los relacionados con la posibilidad de conocer a nuevas personas o el aumento del prestigio deportivo y, una tendencia favorable, en la posibilidad de asistir a un evento internacional. 
Por otro lado, encontraríamos a los residentes denominados como Favorables Moderados, que se trata del grupo más reducido (24\%) y se caracteriza por mostrar una tendencia favorable en muchos de los aspectos preguntados. Este colectivo muestra un elevado apoyo a la celebración del evento, aunque manifiesta cierta neutralidad en aspectos relacionados con el impacto deportivo como la posibilidad de que la celebración del evento proporcione subvenciones y apoyo deportivo a los clubes de la ciudad y, también, en aspectos referidos al impacto cultural tales como el aumento de la cohesión y la unidad de la sociedad gracias a la Fórmula 1.

De este modo la mayor parte de los ciudadanos consultados no se muestran de acuerdo ante la posibilidad de que la Fórmula 1 repercuta a nivel cultural y deportivo en la sociedad. Así, desde el punto de vista del impacto deportivo, se puede comprobar el evidente desacuerdo entre la mayoría de los residentes en la posibilidad de incrementar la práctica deportiva, las instalaciones deportivas, las subvenciones y el apoyo deportivo a los clubes de la ciudad o el hecho de que este pensada para el entretenimiento de los ciudadanos. También, se puede comprobar una clara tendencia al desacuerdo de la mayoría de residentes encuestados con el impacto cultural del evento, destacando los referidos al aumento de la cohesión y la unidad de la sociedad, la oportunidad para conocer a nuevas personas, favorecer la preservación y conservación de la cultura valenciana y la mejora de la solidaridad y la hospitalidad de los ciudadanos con los visitantes.

Aunque en ningún caso esta tendencia es tan acusada en otros estudios similares realizados en otras localidades o regiones sobre eventos deportivos de motor, algunos resultados podrían coincidir con lo detectado por los trabajos de Fredline y Faulkner (2000) y Fredline, Deery y Jago (2005) en los grupos de residentes con una actitud más desfavorable. Sin embargo, otros estudios como el de Zhou (2010) sobre el GP de Macao o el de Cegielski y Mules (2002) sobre la V8 Supercar Race de Camberra (Australia), encontraron que un $77,9 \%$ y un $79 \%$ respaldaban la celebración del evento en sus respectivas localidades. En este trabajo estos porcentajes sólo 
son similares para una cuarta parte de los residentes encuestados que corresponden al grupo más favorable.

En aspectos relacionados con el impacto deportivo, el estudio de Cheng y Jarvis (2010) destacaba que la promoción del deporte era el aspecto menos citado por los residentes de Singapur en relación a la celebración de la Fórmula 1. También, en el trabajo de Añó, Calabuig y Parra (2012) sobre la Fórmula 1 se encontró que los aspectos relacionados con la repercusión deportiva eran los peor valorados. Estos resultados coinciden con lo detectado en este trabajo sobre el impacto deportivo y que puede estar asociado, como ya hemos señalado, a las características propias de este tipo de deportes. En esta línea, en el estudio de Zhou (2010) la mayoría de residentes consideraba que el GP de Macao no proporcionaba instalaciones que pudiesen ser utilizadas posteriormente por los ciudadanos.

Por otra parte, algunos de los aspectos relacionados con el impacto cultural son valorados en otros estudios con una tendencia al desacuerdo aunque de forma menos acusada que en este trabajo. Por ejemplo, en el estudio de Cheng y Jarvis (2010) los ciudadanos no se mostraban de acuerdo sobre la posibilidad de que la Fórmula 1 les permitiese aprender más sobre otras culturas. También, en el estudio de Fredline y Faulkner (2000), la posibilidad de conocer a otras personas obtenía valores diferentes en función del clúster de pertenencia, observándose una tendencia al desacuerdo entre los residentes más desfavorables al evento y la tendencia inversa en el caso de los que manifestaban una actitud más favorable hacia la IndyCar. En cambio, en el trabajo de Zhou (2010) la mayoría de residentes se mostraba de acuerdo ante la posibilidad de que el GP ayudase a los ciudadanos a entender otras culturas o permitiese conocer a nuevas personas.

Estos resultados, muestran las diferencias que existen en función de la población que se analice, ya que cada región o localidad tiene unas características sociales y culturales diferentes. En concreto, en este trabajo hay que tener presente las dificultades socioeconómicas que definen el contexto actual en la región en la que se ha llevado a cabo este estudio, lo que implica una mayor sensibilidad 
de los residentes ante el gasto público efectuado en cualquier acontecimiento. Además, este tipo de eventos requieren de una elevada inversión pública en aspectos relacionados con la construcción, remodelación o adaptación de un circuito de carreras a las normas de la Federación Internacional de Automovilismo (FIA), además de tener que abonar un canon para poder organizar la prueba. En este sentido, estos costes tan elevados, en un contexto socioeconómico desfavorable, podrían explicar la ausencia de un grupo de residentes con una clara actitud favorable hacia el acontecimiento analizado que, por el contrario, sí se ha encontrado en la mayoría de estudios que analizan el impacto en la población local de otros eventos deportivos de motor en diferentes localidades (FREDLINE; FAULKNER, 2000; ZHOU, 2010).

Por otro lado, los resultados de este trabajo contrastan y demuestran lo detectado en estudios previos (CEGIELSKI; MULES, 2002; CHENG; JARVIS, 2010; FREDLINE; FAULKNER, 2000; ZHOU, 2010) en relación a la importancia de contemplar a los residentes como un grupo heterogéneo a la hora de analizar el impacto social de los eventos deportivos, ya que la percepción sobre un evento deportivo puede variar en función del interés, el apoyo, la asistencia o el grado de identificación con el acontecimiento. En este sentido, sería interesante comprobar en qué medida influyen otras variables como los valores socio-políticos, el nivel socio-económico, la proximidad de la residencia al lugar de celebración del evento, el apego hacia la comunidad, el nivel de contacto con el evento, la vinculación laboral o económica con el turismo, el grado de conocimiento del deporte o la participación de los residentes en la planificación del acontecimiento, tal y como se ha analizado en otros trabajos (CEGIELSKI; MULES, 2002; FREDLINE; FAULKNER, 2000; KIM; PETRICK, 2005; WAITT, 2003; ZHOU; AP, 2009). Por último, es necesario seguir evaluando y monitorizando el impacto social de estos acontecimientos, así como, analizar otros eventos para comparar y observar los cambios en las actitudes hacia la acogida de los mismos. 
Análise da percepção dos residentes sobre o impacto cultural e desportivo de um grande prêmio de fórmula 1

Resumo: O objetivo do estudo é analisar a percepção dos residentes em relação ao impacto cultural e desportivo do Grande Prêmio da Europa de Fórmula 1. Realizou-se uma análise cluster, combinando métodos hierárquicos e não hierárquicos, que permitiu encontrar três grupos com percepções diferentes frente a este evento esportivo: Desfavoráveis, Desfavoráveis Moderados e Favoráveis Moderados. Os residentes consideram em sua maioria que este evento não tem um impacto considerável no nível cultural e esportivo. Também se encontraram diferenças entre os três grupos de residentes em função do interesse pela Fórmula 1, o respaldo ao evento, a assistência e o grau de identificação.

Palavras-chave: População residente. Percepção social. Esporte. Fatores culturais.

\section{Analysis of resident's perception on the cultural and sport impact of a formula 1 grand prix \\ Abstract: The aim of this study is to analyze the resident's perception about the cultural and sport impact of European Formula 1 Grand Prix. Cluster analysis was performed by combining hierarchical and non- hierarchical methods, which allowed us to differentiate three groups with different perceptions towards sporting event: Unfavorable, Moderate Unfavorable and Moderate Favorable. Most residents believe that this event has no significant impact to cultural and sporting level. Also, differences were found between the three groups of residents in the interest of Formula 1 , the support for the event, attendance and the degree of identification.}

Keywords: resident population; social perception. Sports. cultural factors 


\section{REFERENCIAS}

AÑÓ, V.; CALABUIG, F.; PARRA, D. Impacto Social de un gran evento deportivo: el Gran Premio de Europa de Fórmula 1. Cultura, Ciencia y Deporte, Murcia, v.7, n. 19, p. 53-65, 2012.

BALDUCK, A.L.; MAES, M.; BUELENS, M. The social impact of the Tour de France: Comparisons of residents' pre-and post-event perceptions. European Sport Management Quarterly, Aachen, v. 11, n. 2, p. 91-113, 2011.

CEGIELSKI, M.; MULES, T. Aspects of residents' perceptions of the GMC 400canberra's V8 supercar race. Current Issues in Tourism, Abingdon, v. 5, n. 1, 54-70, 2002.

CHENG, E.; JARVIS, N. Residents' perception of the social-cultural impacts of the 2008 Formula 1 Singtel Singapore Grand Prix. Event Management, Brisbane, v. 14, n. 2, p. 91-106, 2010.

COALTER, F.; TAYLOR, J. Large Scale Sports Events: Event Impact Framework. Report to UK Sport. University of Stirling, Stirling, v. 23, dic. 2009. Disponible en: http://hdl.handle.net/1893/1942, Acceso en: 25 fev. 2013.

DELAMERE, T.A. Development of a scale to measure resident attitudes toward the social impacts of community festivals, part II: Verification of the scale. Event Management, Brisbane, v. 7, n. 1, p. 25-38, 2001.

FREDLINE, E. Host community reactions to motorsport events: The perception of impact on quality of life. In: RITCHIE, B. W; ADAIR, D. Sport tourism: Interrelationships, impacts and issues. Clevedon (Reino Unido): Channel View Publications, 2004. p. 155-173.

FREDLINE, E.; FAULKNER, B. Host community reactions: A cluster analysis. Annals of Tourism Research, Kidlington, v. 27, n. 3, p. 763-784, 2000.

FREDLINE, E.; DEERY, M.; JAGO, L. Host community perception of the impacts of the Australian Formula One Grand Prix in Melbourne: A comparison of resident reactions in 1999 with 2002. Sustainable Tourism Cooperative Research Centre, Gold Coast (Australia), 2005.

HAIR, J.F. et al. Análisis Multivariante. Madrid: Prentice Hall Iberia, 1999.

KIM, H.J.; GURSOY, D.; LEE, S.B. The impact of the 2002 World Cup on South Korea: Comparisons of pre-and post-games. Tourism Management, Kidlington, v. 27, n. 1, p. 86-96, 2006.

KIM, S.S.; PETRICK, J. F. Residents' perceptions on impacts of the FIFA 2002 World Cup: The case of Seoul as a host city. Tourism Management, Kidlington, v. 26, n. 1, p. 25-38, 2005. 
MIHALIK, J. B.; SIMONETTA, L. A midterm Assessment of the Host Population's Perceptions of the 1996 Summer Olympics: Support, Attendance, Benefits, and Liabilities. Journal of Travel Research, Thousand Oaks, v. 37, n. 3, p. 244-248, 1999.

OHMANN, S.; JONES, I.; WILKES, K. The Perceived Social Impacts of the 2006 Football Cup on Munich Residents. Journal of Sports \& Tourism, London, v. 11, n. 2, p. 129-152, 2006.

PREUSS, H.; SOLBERG, H. A. Attracting major sporting events: The role of local residents. European Sport Management Quarterly, Aachen, v. 6, n. 4, p. 391411, 2006.

SMALL, K.; EDWARDS, D.; SHERIDAN, L. A flexible framework for evaluating the socio-cultural impacts of a (small) festival. International Journal of Event Management Research, Brisbane, v. 1, n. 1, p. 66-77, 2005.

TAVARES, O. Megaeventos esportivos. Movimento, Porto Alegre, v.17, n. 3, p. 11-35, 2011.

WAITT, G. Social impacts of the Sydney Olympics. Annals of Tourism Research, Kidlington, v. 30, n. 1, p. 194-215, 2003.

WEED, M.; COREN, E.; FIORE, J. A Systematic Review of the Evidence Base For Developing a Physical Activity and Health Legacy From the London 2012 Olympic and Paralympic Games. Canterbury: SPEAR, 2009.

ZHOU, J.Y. Resident perceptions toward the impacts of the Macao Grand Prix. Journal of Convention \& Event Tourism, Philadelphia, v. 11, n. 2, p. 138-153, 2010.

ZHOU, J. Y.; AP, J. Residents' perceptions towards the impacts of the Beijing 2008 Olympic Games. Journal of Travel Research, Thousand Oaks, v. 48, n. 1, p. 7891, 2009.

Endereço para correspondência:

Ferran Calabuig Moreno

Departament Physical Eduaction and Sports

Gascó Oliag, 3

46010 Valencia (España)

Recebido em: 31.05.2013

Aprovado em: 23.10.2013 